\title{
Hacia una autonomía relacional: movimientos culturales urbanos contemporáneos
}

\author{
MARÍA TERESA ANGUlo DelGado \\ Doctorando FPU. Departamento de Dibujo I. Facultad de Bellas Artes \\ Universidad Complutense de Madrid \\ maite.angulo.delgado@gmail.com \\ CARLOS MARTÍNEZ-ARRARÁS CARO \\ Profesor Colaborador. Departamento de Teoría y Proyectos en Arquitectura y Urbanismo \\ Escuela Politécnica Superior / Universidad CEU San Pablo \\ martinezarraras@gmail.com \\ ANTONIO RABAZAS ROMERO \\ Profesor Titular. Departamento de Dibujo I. Facultad de Bellas Artes \\ Universidad Complutense de Madrid \\ arabazas@art.ucm.es \\ DENICA VeSELINOVA SABEVA \\ Doctorando. Departamento de Dibujo I. Facultad de Bellas Artes \\ Universidad Complutense de Madrid \\ denica.veselinova@gmail.com
}

\begin{abstract}
Resumen
Desde finales del siglo XVII hasta nuestros días la idea de la autonomía del arte y su búsqueda de emancipación con respecto a las autoridades respectivas en cada momento histórico ha vivido una larga e intensa evolución, plagada de logros y fracasos, que sigue viva hoy en día espoleando la actividad cultural y artística en nuestras ciudades. En la época de la Ilustración se buscó la autodeterminación del individuo más allá del poder y el control del Estado absolutista consiguiendo desligar el ámbito de la estética de pretextos teológicos. Más adelante la modernidad se centró en denunciar y combatir el ambiente de hastío y falsa moral de la sociedad burguesa instituida interfiriendo en la noción de normalidad imperante y su tendencia unificadora. Sin embargo, todo el potencial revulsivo de la primera formulación de la autonomía desapareció y las prácticas artísticas dejaron de tener implicaciones sociopolíticas, convirtiéndose en un mero ornamento de buen gusto. Desde entonces hasta ahora han
\end{abstract}


sido muchos los movimientos que se han esforzado en proponer otra cultura alternativa a la dominante, lidiando y negociando a la vez con la vulgaridad y la invasión de la cultura de masas y con el voraz mercado del arte. Este trabajo, ligado al grupo de investigación Derivas Urbanas. Intervenciones artísticas en el territorio, pretende descubrir y poner en valor prácticas artísticas actuales vinculadas a la tradición de movimientos contraculturales. A través de diferentes estrategias como la ocupación, la auto-gestión o la co-gestión, se promueve un debate crítico en torno a cuestiones como la función del espacio público o la capacidad y el derecho de los ciudadanos a una participación activa en la producción, difusión y recepción de la cultura, contribuyendo de esta manera a la definición y articulación de una estética relacional.

Palabras clave: Estética relacional, autonomía del arte, auto-gestión, participación, contracultura.

\begin{abstract}
From the late seventeenth century to the present day the idea of the autonomy of art and its pursuit of emancipation from the authorities in each historical period has gone through a long and intense evolution, with plenty of successes and failures, which is still alive today spurring the cultural and artistic activity in our cities. During the Enlightenment self-determination of the individual was sought beyond the power and control of the Absolutist State and the field of aesthetics was finally decoupled from theological purposes. Later on modernity focused on exposing and fighting a certain atmosphere of boredom and false morality typical of the bourgeoisie by interfering with the prevailing notion of normality and its unifying tendency. However, all the stimulating potential of the first formulation of autonomy disappeared and artistic practices ceased to have sociopolitical implications, becoming a mere and tasteful ornament. From then until now many movements have struggled to propose an alternative to the prevailing culture, dealing and negotiating simultaneously with the vulgarity and the invasion of mass culture and the voracity of the art market. This work, linked to the research group Urban Derives. Artistic interventions in the territory, intends to discover and add value to current artistic practices connected to the tradition of countercultural movements. Strategies such as occupation, self-management or co-management are employed to promote critical debate around issues such as the function of public space or the ability and the right of citizens to actively participate in the production, diffusion and reception of culture. This way it is provided a contribution to the definition and articulation of relational aesthetics.
\end{abstract}

Key words: Relational aesthetics, autonomy of art, self-management, participation, counterculture. 
Sumario: 1.- Introducción. 2.- De la autonomía ilustrada a la autonomía moderna. 3.Desde la utopía hacia una autonomía relacional. 4.- ¿Qué entendemos por relacional? 5.- El caso del Campo de Cebada. 6.- Conclusión. 7.- Referencias bibliográficas.

\section{Introducción.}

La posición material y espiritual del arte es tan desesperada que no debería esperarse del pintor que fuera complaciente cuando pinta. Dejemos la complacencia a la institución (...) El arte no tiene nada que ver con la verdad. La verdad se encuentra en los intersticios. Pretender ser objetivo es ser sectorial y ser sectorial es pedante y aburrido. (...) El arte es la montaña de estiércol en que crece el kitsch. (...) En vez de un idealismo abstracto, lo que buscamos es un nihilismo honesto (Home, 2004: 90).

Este fragmento pertenece a un texto publicado por la revista Spur en 1958 y es una de tantas manifestaciones que dan cuenta de los reveses del arte y su delicada relación con el ámbito de la verdad o el conocimiento.

La creencia de que todo conocimiento humano está ligado a la praxis fue uno de los pilares fundamentales de la filosofía clásica. Todos los individuos, ya se dedicaran a la artesanía, la medicina o las artes militares, necesitaban del conocimiento para desempeñar su función adecuadamente y mantener su forma de vida, y con ello la posibilidad de su felicidad. Sin embargo el conocimiento fue jerarquizado según una escala de valores que separaba los saberes funcionales necesarios para la vida cotidiana, es decir, lo útil y necesario, del conocimiento filosófico desinteresado, lo bello y placentero, situado muy por encima y encargado de la felicidad de los hombres. De esta manera, la filosofía se convirtió en un arte superior destinado a una élite encargada de revelar las verdades supremas, mientras que la lucha por la existencia material y todos los saberes relacionados con ella, se enfrentaban a la contingencia, la miseria y la explotación. Herbert Marcuse sitúa la quiebra del equilibrio entre praxis, conocimiento y felicidad en este preciso momento y apunta que fue el temor a la inestabilidad, la anarquía y las debilidades del ser humano lo que llevó a la filosofía a situar las cuestiones referidas al conocimiento, la bondad y la verdad en un plano idílico, confinando por tanto la libertad y la felicidad del individuo, en un ámbito trascendente, más allá de la facticidad de los hechos (Marcuse, 2011: 6). 
Desde que el ámbito de lo estético se independizó, y la experiencia estética se convirtió en un fin en sí mismo, no supeditada ni al Estado ni a la Razón, la función del arte ha oscilado entre lo decorativo y lo metafísico, lo social y lo político, pero ha estado siempre inscrita en un sistema sociopolítico determinado y condicionada, de una u otra manera, por el mercado.

El debate sobre la función social del arte ha acompañado y guiado muchas de las manifestaciones artísticas del siglo $X X$, que han inventado y reinventado estrategias amparadas en el extrañamiento o sublimación de la obra de arte, en la exploración de lo artificial, lo irracional y lo artesanal o en la disolución del arte en la vida cotidiana y en la pura acción política. Pero todos los esfuerzos se han topado obstinadamente con dos grandes impedimentos: por un lado, el mercado del arte y su invasión en los esquemas conductuales y relacionales de las personas, y por otro lado, el poder y su empleo de la sensibilidad para legitimar determinadas ideologías.

En este trabajo vamos a realizar un breve recorrido de la idea de la autonomía del arte deteniéndonos en tres momentos decisivos, la Ilustración, la modernidad y la época actual, y en un conjunto de prácticas artísticas que, del futurismo al situacionismo y de las actividades fluxus a los actuales movimientos urbanos de reivindicación del espacio público, podrían caracterizarse por la búsqueda de una cierta restauración de la totalidad y por la defensa de la autodeterminación del individuo a través de la experiencia estética.

\section{De la autonomía ilustrada a la autonomía moderna.}

Uno de los mayores logros de la filosofía de la Ilustración fue la búsqueda y definición de la autodeterminación de los individuos más allá del control y el poder absolutistas. Se apoyaron en la creencia de que todo ser, incluido el ser humano, tiene un principio dinamizador, una thatkraft o fuerza activa, según Moritz, que le hace desarrollarse según patrones internos, al margen de todo interés o fin externo a sí mismo. Surge entonces el concepto de un sujeto autodeterminado, responsable único de su propia felicidad, que ha de determinar sus propios objetivos y fines y la manera de satisfacer sus necesidades.

Por otro lado, desde finales del siglo XVIII y comienzos del siglo XIX hubo un proceso de erosión de uno de los supuestos básicos del clasicismo: la idea 
de una belleza universal e intemporal. La universalidad no se encuentra en el objeto, que es contingente, ni en el sujeto, que es particular, sino en la propia estructura perceptiva, en la capacidad común de todos los hombres de disfrutar de la belleza, en una suerte de solidaridad universal que Kant denominó sensus communis, y que predispondría a los individuos hacia una armonía (Kant, 2007: 154). El ámbito del gusto se desvincula, pues, de todo interés, fin o concepto, no se rige ni por la razón ni por la moral, sino que está relacionado con la interioridad de cada uno, lo que supone un atentado contra la tradición academicista del siglo XVII.

Gracias a esta recién conquistada autonomía del gusto la clase media ve la posibilidad de cultivar un gusto personal que no esté supeditado a un orden racional encabezado por la obediencia al rey. Este gusto afecta a la moda, el diseño de los jardines, la decoración de los hogares e incluso a la personalidad misma. La actitud, la pose y el comportamiento social se llenan de valores estéticos en busca de la singularidad. La autonomía que en un principio sólo se refería a un concepto y uso de lo estético desligado de la Corte y la Iglesia, va poco a poco afectando a otros ámbitos de discusión de carácter más político. A través de una sucesión de encuentros en cafés, salones y tabernas en los que participaban personas de diferentes clases sociales, se va tejiendo una socialidad en la que se suspenden temporalmente las jerarquías. En este momento, la burguesía, aunque sostenida por el Estado, comenzó a concebirse como no dependiente de él.

A medida que avanza el siglo XIX, el pensamiento evoluciona desde una estética que imita la naturaleza hacia una estética cada vez más alejada de la realidad. La naturaleza ha dejado de ser idílica y se ha vuelto egoísta, violenta y destructiva. El romanticismo convierte lo bueno, lo bello y lo verdadero en ideales que están más allá de las condiciones de vida existentes.

Cuando lo bello atrae hacia sí nuestra contemplación, la sustrae un tiempo de nosotros mismos y hace que nos parezca que nos perdemos en el objeto bello; y precisamente esta pérdida, este olvido de nosotros es el más alto grado de goce puro y desinteresado que proporciona lo bello. Sacrificamos en un momento nuestra existencia individual y limitada en aras de una instancia superior (Claramonte, 2010: 48).

La cultura se universaliza y deja de considerarse patrimonio de unos pocos. De esta forma, la belleza de una obra de arte debe afectar, obligar y agra- 
dar a todos por igual. La cultura se impone como una forma de iluminar y penetrar la existencia de los hombres, que han de aprehender los valores culturales e incorporarlos a su vida. La clase media recoge el legado de Schiller o Rousseau y se otorga la tarea de educar e iluminar a las masas a través del arte y el buen gusto. Se desarrolla así una forma de poder o de autolegitimación que apela a la sensibilidad para vehicular determinados valores morales.

En cualquier caso, esta igualdad y universalidad abstractas con respecto al acceso a la cultura y a la capacidad de disfrutar de ella se convierte en desigualdad concreta ya que gran parte de la población no tiene siquiera acceso a la satisfacción de las necesidades más elementales. De este modo surge lo que Marcuse denomina cultura afirmativa, que define como un concepto abstraído de una (falsa) totalidad social, elevado a la categoría de (falso) patrimonio colectivo y (falsa) universalidad (Marcuse, 2011: 13).

La autonomía ilustrada logró frenar cierto tipo de atropello a la naturaleza o a determinadas clases sociales, mediante el despertar de una conciencia de autodeterminación, pero esta conciencia siempre se utilizó en favor del lucimiento de la burguesía y de la nobleza y, sobre todo, a favor de los planes políticos del Estado. Se trataba de una autonomía tolerada siempre y cuando permaneciera en un ámbito meramente simbólico y no pasara a la acción o interfiriera en la vida política. Razonad cuanto queráis y sobre lo que queráis, pero obedeced. Tal era la máxima de Federico el Grande (Claramonte, 2010: 42).

No obstante, Marcuse rescata una consecuencia positiva del idealismo burgués: la capacidad revolucionaria y de transformación que se despierta en el individuo al experimentar lo que no se tiene. El arte burgués alejó tanto sus formas ideales del acontecer de la vida cotidiana, que el individuo se hizo consciente de que su felicidad sólo podría venir dada tras la desaparición de ese mundo. La teoría estética en la temprana sociedad capitalista fue radical al considerar las energías humanas como fines en sí mismos y enemigas pues de cualquier tipo de dominación (Eagleton, 2006: 60).

Cuando el arte y su pequeña esfera pública asumen que la recién conquistada autonomía ha sido institucionalizada y su potencial revulsivo neutralizado, y que lo único que se ha conseguido es la legitimación de la burguesía y de un conjunto de valores y convenciones ligados a ella, comienza a gestarse un movimiento de autorechazo y negación. El individuo, si bien es libre en la 
economía de mercado o la democracia representativa, está sujeto por imperativos internalizados de autocontrol. Al arte no le queda sino negar y rechazar la normalidad burguesa, y lo hacen explorando los caminos de lo irracional, lo artificial, lo primitivo, lo anormal o lo perverso. Se pasa también a invadir lo que hasta entonces, en aras de la distinción y la inmunidad, se había evitado a toda costa: la vida cotidiana.

Las vanguardias pensaron que la acción revolucionaria en su práctica artística y su ataque o alteración de los modelos del arte equivaldría a la acción en la vida política y la alteración del orden público. Pero los caminos que siguieron acabaron enfrentados a un abismo. Algunos se olvidaron de sus reivindicaciones encerrándose en la autorreferencialidad, otros prescindieron de la formalización de sus ideas diluyéndose en la pura acción subversiva y fanfarrona o en las vicisitudes de la vida cotidiana.

Sin embargo, la radicalidad del rechazo vanguardista a la sociedad y al sistema del arte y las diversas tentativas de acercamiento a la vida cotidiana tuvieron una gran influencia en todos los movimientos posteriores que a lo largo del siglo XX se han esforzado por la búsqueda de una cultura alternativa a la oficial, más arraigada a las condiciones materiales de la vida y dotada de una naturaleza transformadora.

\section{Desde la utopía hacia una autonomía relacional.}

Las vanguardias dejaron tras de sí una lección. El mercado del arte era un enemigo mucho más poderoso y persuasivo de lo que parecía. Una cosa es que la acumulación de negatividad ayude a la crítica social y política, y otra que se convierta en un fin en sí mismo (Claramonte, 2010: 190). De cualquier manera, movimientos posteriores que ahora vamos a analizar recogerán el legado de las vanguardias y continuarán en su afán de autonomía desplegando estrategias, un tanto utópicas, para lidiar con la avidez el mercado o la dominación de las ideologías políticas, teniendo siempre en mente la posibilidad de la existencia de una cultura verdaderamente "popular".

En 1948 tras un congreso sobre arte de vanguardia, un grupo de artistas provenientes de Dinamarca, Bélgica y Holanda, enfurecido por el tono complaciente del mismo, decide marcharse y constituirse como colectivo, deno- 
minándose COBRA. Críticos con el surrealismo, la abstracción y el realismo soviético, abogan por un arte popular colectivo y directo. Constant, uno de sus integrantes, escribiría en un texto titulado Declaración de libertad:

En el vacío cultural sin precedentes que ha seguido a la guerra (...) cuando la clase dominante ha llevado el arte a una posición de total dependencia (...) se ha establecido una cultura del individualismo condenada por la misma cultura que la ha producido porque su convencionalismo impide el ejercicio de la imaginación, el deseo y la expresión de la vida (...) En tanto las formas artísticas sean una imposición histórica, no podrá haber un arte popular, ni siquiera cuando se hacen concesiones al público mediante la participación activa. El arte popular se caracteriza por expresar vida de un modo directo y colectivo (Home, 2004: 44).

Por otro lado, integrantes del Movimiento Letrista se esforzaban por recuperar el carácter "expansivo" de la cultura tras la tendencia que Isou denominaba "desbastadora", que había llevado de lo narrativo a lo anecdótico con Baudelaire, de lo anecdótico a la rima y las palabras con Rimbaud y de ahí al espacio y el sonido con Mallarmé. Si bien no realizaron una oposición radical a la alta cultura, sino que más bien, la veían necesaria, ni una crítica materialista a la sociedad, se dedicaron a teorizar acerca de todos los aspectos de la vida:

No es nuestro propósito crear una sociedad socialista o comunista al uso, en que los hombres trabajen a cambio de recompensas abundantes y estáticas. Sino que propugnamos avanzar hacia una sociedad ideal en que los hombres vivan mucho más, al reducir la maldición del trabajo al mínimo, y aspiren a un gozo pleno y a un éxtasis progresivo (Home, 2004: 55).

Dentro de este grupo se produjo una escisión radical, la Internacional Letrista que en vez de producir objetos de arte se dedicó a vivir la revolución a través de una exploración sensorial de la ciudad, lo cual desembocaría más tarde en la Internacional Situacionista, el urbanismo unitario y la teoría de la deriva. En ese momento aparece Guy Debord, quien disgustado por la evolución errónea del surrealismo y su desvío a través de los caminos del inconsciente, plantea la elaboración de un programa cultural de emancipación del espectador, y busca la ruptura de la identificación psicológica del espectador con el héroe, para hacerle consciente de la alienación en la que vive inmerso, y arrastrarle a la acción y la revolución. Tras un congreso de una semana en las montañas del norte de Italia en el que convergen colectivos y artistas de 
distintos lugares, se crea en 1957 la Internacional Situacionista, cuyo bloque teórico y organizativo estaba constituido por Constant, Jorn, Gallizio, Bernstein y su marido Debord. Jorn y Constant estaban a favor de una actividad colectiva, no competitiva y lúdica que desarrollaba mediante derivas, mapas psicogeográficos y creación de situaciones. Debord y Bernstein sin embargo optaban directamente por un abandono total del arte. Esta escisión entre la sección cultural y la sección política del movimiento causó la creación de la II Internacional Situacionista en 1962.

$\mathrm{Al}$ otro lado del Atlántico, comienza a surgir otro grupo que no se dedica tanto a la exploración sensorial de la ciudad o a justificaciones teóricas que realicen el arte a través de su supresión o lo supriman a través de su realización. Más bien, el objetivo consiste en sabotear las actividades de la alta cultura $-\mathrm{y}$ de paso la vida cotidiana de los ciudadanos- a través de una estética de la sencillez que todo el mundo puede replicar. Las acciones de Fluxus eran de naturaleza destructiva y extravagante. Interferían en el sistema de comunicaciones de la ciudad, creando situaciones de caos y desconcierto, manipulaban objetos y sonidos cotidianos y vulgares, tanto artificiales como orgánicos, explorado los límites entre lo vulgar y lo refinado. Su sentido de participación no consistía solamente en incluir a la audiencia directamente en sus acciones, sino en el uso de guiones que cualquiera pudiera interpretar sin necesidad de práctica, habilidad o preparación. La estética fluxus era intencionalmente una forma de asalto a la cultura seria. De cualquier manera, la falta de consenso entre los integrantes más radicales y aquellos que tenían una actitud algo más negociadora con la institución, unido al ambiente hippie de finales de los sesenta y al carácter complaciente y sensual de las actividades que se organizaban, hizo que el tono crítico y radical del primer fluxus se diluyera lentamente.

Fluxus fue el ejemplo más evidente de un movimiento de la producción de objetos de arte hacia los dominios de lo no artístico, pero desde luego no el único. Ray Johnson, padre fundador del mail art, nunca perteneció a Fluxus, pero su estética es muy cercana a estos. Adoptó el nombre de The Ney York Correspondance School y sus actividades consistían principalmente en el envío de cartas y dibujos a familiares y amigos. Poco a poco fue generando una extensa red de correo postal. Con el desarrollo del arte conceptual y la performance, cuya forma de documentación consistía en notas y fotografías, aumentó la cantidad de gente que intercambiaba ideas y rarezas por correo pos- 
tal. A principios de los 70, se hicieron y publicaron listas de direcciones para gente interesada en intercambiar ideas y trabajos. Este tipo de prácticas que en un principio desarrollaban unos cientos de personas acabó atrayendo a miles, y así se fue generando una "nueva forma cultural" (Home, 2004: 149).

Otra forma de asalto a la alta cultura y a la mercantilización del arte fue la desarrollada por Gustave Metzger a través de su "estética de la revulsión", que implicaba un arte autodestructivo, financiado por el Estado, como sustituto de la guerra o del instinto de aniquilación del hombre, y como única forma de luchar contra la mercantilización del arte y el rol del artista-geniocreador. También nos parece pertinente sacar a relucir las actividades del colectivo holandés Provo y sus planes blancos que consistían en atacar el concepto de propiedad privada defendido por el capitalismo, disponiendo por ejemplo bicicletas sin candado en la calle para uso público, que la policía rápidamente incautaba. Este grupo consiguió la atención de la comunidad médica con la acción de Bart Huges, quien se hizo una trepanación con la intención de expandir sus capacidades perceptivas.

Las prácticas mencionadas son sólo algunas de las muchas que se han llevado a cabo a lo largo del siglo pasado en este contexto de oposición al capitalismo cultural. Gracias a su ingenuidad, provocación y naturaleza lúdica atrajeron la atención de muchas personas críticas con el sistema capitalista y la mercantilización de la cultura, pero llamaron también la atención del propio sistema, que supo no sólo anular el carácter reivindicativo de estas propuestas, sino aprovecharlo e incluirlo en su modus operandi, convirtiendo en objeto de consumo toda manifestación artística y política.

\section{4. ¿Qué entendemos por relacional?}

Después de este pequeño repaso histórico de algunas de las prácticas culturales que han tratado de buscar en el arte un espacio de libertad y autonomía -no sólo con el objetivo de encontrar las condiciones óptimas para crear en libertad, sino, sobre todo, con el objetivo de recuperar una función social del arte no sujeta a las leyes de la monarquía, la religión, la burguesía o el mercado- y asumiendo que, a pesar de sus pequeños logros, ninguna de ellas ha conseguido el objetivo último, nos hacemos la siguiente pregunta: ¿Y ahora qué? ¿Qué respuestas se han dado en el mundo del arte en los últimos años? ¿Qué está pasando ahora? 
Somos conscientes de que es una pregunta muy difícil de responder, pero vamos a tratar de dar algunas claves, si no para encontrar una solución mágica, por lo menos para entender qué está pasando ahora y por qué parece estar tan de moda lo que en su día Nicolas Bourriaud denominó estética relacional (Bourriaud, 2008: 9).

Desde los años noventa hasta ahora ha aumentado considerablemente el número de artistas que organizan fiestas, cenas, encuentros y talleres en museos, instituciones y galerías, haciendo gran hincapié en trasladar el interés desde el objeto de arte a la experiencia social compartida entre los espectadores o participantes, y promover con ello un cierto tipo de cohesión social. Realizan acciones como, por ejemplo, colocar chimeneas reales dentro de las salas de exposición, asunto que imaginamos será de gran regocijo para los vigilantes de sala, pero que tiene escasa utilidad para aquellos que realmente necesitan de ese calor y no pueden acceder a él pasada la hora de cierre de la institución, si es que en algún momento se les ha permitido el acceso. En cualquier caso, esta dematerialización de la forma artística ha sido investigada mucho antes de los noventa desde el ámbito del arte conceptual o la performance, por poner un ejemplo. Una novedad no es por lo tanto. Por otro lado, la función socializadora del arte es inherente a toda experiencia estética, sea de la índole que sea y más allá de que el autor lo haga explícito en el despliegue formal de su propuesta o en la obstinación por prescindir totalmente de la forma.

A pesar de todo, este tipo de prácticas existen, se siguen perpetuando $y$, aunque disten mucho de lograr sus objetivos, promueven un cierto debate que, pensamos, beneficia más a las instituciones que acogen las propuestas que a los sujetos que se ven involucrados en ellas.

La obra de Michel de Certeau La invención de lo cotidiano, un análisis antropológico de los hábitos de consumo contemporáneos, ofrece una visión poco común, por su carácter esperanzador, acerca de la influencia del capitalismo contemporáneo en la sociedad. Contrariamente a las tesis que se limitan a la consideración de un sujeto inevitablemente sometido al poder del estado, de Certeau ve en el individuo contemporáneo a un consumidor potencialmente activo y creativo, dotado de un gran arsenal de "micro-resistencias" en forma de tácticas sutiles y silenciosas imbricadas con la vida cotidiana y capaces de generar lo que él denomina "micro-libertades" (De Certeau, 2000). 
A continuación vamos a hablar de un proyecto que bascula entre los hábitos de consumo creativos teorizados por De Certeau y lo que entendemos por arte de contexto (Claramonte, 2011: 18). Nos interesa examinar no tanto lo que este tipo de prácticas fabrican o el contexto en el que se desarrollan, sino la forma en que actúan, las relaciones que despliegan y los cambios significativos que insinúan.

\section{El caso del Campo de Cebada.}

En agosto de 2009 el solar que albergaba el antiguo polideportivo del barrio de la Latina fue derruido debido a un Plan de Rehabilitación del Centro Urbano que afectaba también al contiguo Mercado de la Cebada, proyecto que lleva paralizado más de tres años debido a la falta de liquidez del Estado. Un año más tarde el solar abrió sus puertas durante veinte días para acoger la iniciativa "Isla Ciudad", llevada a cabo por el colectivo francés Exyzt, promocionada por Mahou y promovida por la Casa Encendida. Este evento causó gran revuelo en el vecindario e hizo que un conjunto de personas, colectivos y asociaciones se reunieran para solicitar al Ayuntamiento de Madrid la apertura y cesión del solar, así como una pequeña ayuda económica para habilitar el espacio. De esta manera surge el proyecto El Campo de Cebada (http: //elcampodecebada.org), iniciativa vecinal para la reactivación temporal del espacio urbano que ocupa el solar, espacio que actualmente funciona como una plaza pública equipada en la que se puede jugar al baloncesto, disfrutar del cine, el teatro o la música, plantar tomates y berenjenas o conversar y descansar.

Este proyecto nos parece interesante porque recoge el legado de toda una tradición de movimientos que se han dirigido en busca de la revinculación del arte con los procesos normales de la vida cotidiana, la expansión de la cultura hacia otras esferas del conocimiento y la participación directa de la población en la generación de una cultura popular. En este espacio se promueve una expansión de las capacidades de percepción, creación y acción del individuo, a través de una variada propuesta de actividades culturales que no requieren de una trepanación del cerebro a lo Provo, sino de una participación facultativa. Por otra parte, las dinámicas que se generan se traducen en una socialidad horizontal y una forma de comunidad orgánica, en el sentido en 
que la entendía Ferdinand Tönnies, es decir, como una vida en común, duradera y auténtica, contrapuesta a la naturaleza ideal y mecánica de la sociedad (Tönnies, 1947: 21).

El Campo de Cebada, lejos de funcionar a base de la gran inauguración y la gran clausura, interfiere en la vida cotidiana de las personas que se involucran en él, proponiendo actividades constantemente y abriendo sus puertas prácticamente todos los días. Sigue un modelo replicable, como propusiera el programa de fluxus o del mail-art, que de hecho se puede observar actualmente en distintos espacios ocupados, autogestionados o co-gestionados, tanto del centro de Madrid como de la periferia, a pesar de las especificidades de cada uno de ellos. En ese sentido, hay un esfuerzo por generar un archivo y una documentación, no con un sentido nostálgico de querer retener un tiempo ya pasado, sino más bien como una estrategia de análisis y transmisión de la experiencia. Es un proyecto generativo y expansivo en el sentido en que se gesta una cultura popular de forma directa y colectiva, como en su momento clamaban grupos como COBRA o el Movimiento Letrista. Existe una articulación entre lo artístico, lo social y lo político, en la medida en que hay una negociación entre agentes de la Junta Municipal del Distrito Centro o Participación Ciudadana, colectivos de arte y arquitectura, asociaciones de distinta índole y la comunidad de vecinos. Está situado en el tiempo y en el espacio actual, es más, no lo estará por mucho tiempo, puesto que es de esperar que en algún momento se construya el proyectado polideportivo. Existe una socialidad horizontal, todas las decisiones se toman de forma asamblearia, y los recursos obtenidos se destinan a usos comunes. Por último, no tiene una función unívoca sino que satisface paralelamente distintas necesidades que tienen que ver con la identidad, el ocio, la creación, la libertad, la participación, el entendimiento o el afecto.

Ahora bien, todas las características mencionadas hasta ahora podrían referirse a cualquier centro sociocultural al uso o, por lo menos, a alguno que otro. ¿Cuáles serían las particularidades de este espacio en concreto?

Primero, el espacio funciona con la lógica del hacer. Si no se hace nada, el solar está cerrado, lo que fomenta la iniciativa. Pero además se trata de un hacer colectivo, que atañe no sólo a la propuesta de actividades y usos, sino a una variedad de funciones que van desde la limpieza y adecuación del espa- 
cio a la apertura y cierre de puertas. Todo el que quiera puede proponer un proyecto, que se debate en asamblea atendiendo, más que a criterios de calidad, a cuestiones de agenda y calendario. Las asambleas, además, permiten resolver los conflictos, ya sean de carácter práctico o ideológico, mediante el diálogo y la puesta en común.

Segundo, se trabaja con unos tiempos abiertos y flexibles. Por ejemplo, durante el tiempo de habilitación del campo y del mobiliario urbano construido, se pudo habitar el espacio, haciéndose evidentes determinadas necesidades a lo largo del proceso, y modificando y creando en función de ellas. Esta dinámica de rehabilitación, transformación y adaptación es de hecho un proceso continuo.

Tercero, el solar consta de una serie de características que permiten llevar las rutinas de lo doméstico a la calle, desdibujando y complejizando de esta manera las líneas que separan lo público de lo íntimo.

Cuarto, bajo el conjunto de prácticas que se lleva a cabo en el solar subyace una lógica conjuntiva (basada en la conjunción de propuestas multifuncionales) y no disyuntiva (basada en la separación progresiva de tiempos y lugares) y resultan por tanto restauradoras de unión.

\section{Conclusión}

La filosofía ilustrada habla de la autonomía como base de la dignidad de todo individuo, pero sólo en tanto libertad de pensamiento, no de acción. Kant pensaba que progresivamente la libertad de pensamiento fermentaría y se convertiría en libertad de acción. Dos siglos y medio después, el debate sobre la autonomía sigue afectando a las políticas culturales, y la búsqueda de una libertad de acción a través de la experiencia estética continúa guiando la práctica artística y cultural.

A nuestro modo de ver, iniciativas urbanas como el proyecto del Campo de Cebada, retoman la idea introducida al comienzo sobre la quiebra del equilibrio entre conocimiento, praxis y felicidad, convirtiendo el miedo a la contingencia y a la vulnerabilidad humana en fuente de alianza y solidaridad, y transformando la conciencia de la dificultad y de la contradicción en necesidad de ilusión y posibilidad de cambio. 
Claramonte (2011: 116-117) apunta una de las posibles líneas de investigación a seguir con el fin de dilucidar lo que él denomina autonomía modal. La clave estaría en la propuesta de prácticas que articularan usos estratégicos (en este caso, una negociación a largo plazo con el poder) y usos tácticos, (como por ejemplo un rechazo tajante a determinadas imposiciones totalitarias que, en este caso, tienen que ver con la producción, distribución y recepción cultural, y con los usos y funciones del espacio público).

El arte es algo intelectual y abstracto pero hunde sus raíces en la experiencia cotidiana, en lo humano y lo concreto. A principios del siglo XX John Dewey señalaba:

(...) se impone una tarea para el que pretende escribir sobre la filosofía de las bellas artes. Esta tarea consiste en restaurar la continuidad entre las formas refinadas e intensas de la experiencia que son las obras de arte, y los acontecimientos, hechos y sufrimientos diarios, que se reconocen universalmente como constitutivos de la experiencia. (Dewey, 2008: 4).

No obstante, hemos visto como el acercamiento del arte a la sensibilidad de la vida cotidiana se ha convertido en una trampa más que en una salida airosa, convirtiendo el idealismo, el esteticismo o especialización en armas de doble filo. Por ello conviene no olvidar el potencial político de la forma artística como tal y la necesidad de un cierto extrañamiento, tal y como lo entendía Marcuse:

(...) en contraste con la estética marxista ortodoxa reconozco el potencial político en el propio arte, en la forma estética como tal. Es más, sostengo que en virtud de su particular forma estética el arte es en gran medida autónomo frente a las relaciones sociales dadas. En su autonomía, el arte tanto manifiesta su denuncia ante tales relaciones como a su vez las trasciende. Por esa razón el arte subvierte la consciencia dominante, la experiencia normal. (Marcuse, 2007:53)

\section{Bibliografía}

BOURRIAUD, Nicolas (2008): Estética relacional, Adriana Hidalgo, Buenos Aires. CLARAMONTE, Jordi (2010): La república de los fines, CENDEAC, Murcia.

ClarAmONTE, Jordi (2011): Arte de contexto, Nerea, San Sebastián.

DE CERTEAU, Michel (2000): La invención de lo cotidiano, Universidad Iberoamericana, Instituto Tecnológico y de estudios superiores de Occidente, México. 
DEWEY, John (2008): El arte como experiencia, Paidós Ibérica, Barcelona.

EAGLETON, Terry (2006): Ideología de la estética, Trotta, Madrid.

HOME, Steward (2004): El asalto a la cultura, Virus, Barcelona.

KANT, Immanuel (2007): Crítica del Juicio, Tecnos, Madrid.

MARCUSE, Herbert (2007): La dimensión estética, Biblioteca Nueva, Madrid.

MARCUSE, Herbert (2011): El carácter afirmativo de la cultura, El Cuenco de Plata, Buenos Aires.

TÖNNIES, Ferdinand (1947): Comunidad y sociedad, Losada, Buenos Aires.

El proyecto El Campo de Cebada. Madrid, disponible en:

http://elcampodecebada.org/?page_id=35 (Acceso 20 de noviembre de 2012) 